\title{
$90 \mathrm{Ma}$ 以来热点与西南印度洋中脊的交互作用: 海台与板内海山的形成
}

\author{
张涛 (1)(2)(4)* ${ }^{\text {林间 }}{ }^{(5)}$ ，高金耀 ${ }^{(3)(4)}$ \\ (1) 中国科学院测量与地球物理研究所, 武汉 430077; \\ (2) 中国科学院研究生院, 北京 100049 ; \\ (3) 国家海洋局第二海洋研究所, 杭州 310012; \\ (4) 国家海洋局海底科学重点实验室, 杭州 310012; \\ (5) 美国伍兹霍尔海洋研究所地质地球物理系, 美国马萨诸塞州伍兹霍尔 02543 \\ *E-mail: zhangtaosio@gmail.com
}

收稿日期: 2010-01-13; 接受日期: 2010-10-14

国家海洋局青年海洋科学基金(编号: 1084-10)和基本科研业务费专项资金(编号: JG0706)资助

\begin{abstract}
摘要本文研究了西南印度洋底地幔热点-洋中脊交互作用与海台、海山形成的关系. 先利 用板块重构确定了西南印度洋区域中热点与洋中脊相对位置及海台、海山的形成年代, 然后 通过水深异常和艾里均衡模式计算了相应热点的岩浆熔融通量. 计算结果显示, 自 $90 \mathrm{Ma}$ 以 来, 马里昂(Marion)热点的活动可分为三个阶段: 与古罗德里格斯(Rodrigues) 三联点相互作 用阶段(90 73.6 Ma)、与西南印度洋中脊相互作用阶段(73.6 42.7 Ma)及板内火山活动阶段 (42.7 0 Ma). 这三个阶段分别对应于德尔卡诺隆起(Del Cano Rise)的东部、中部和西部区域 洋底海台的形成. 马里昂热点的活动强度和周期性明显受到了热点离洋中脊距离的影响. 马 里昂热点的活动周期约为 $25 \mathrm{Ma}$, 长过夏威夷和冰岛热点的活动周期(约 $15 \mathrm{Ma}$ ).
\end{abstract}

关键词

西南印度洋

地幔热点

洋中脊

交互作用

岩浆熔融通量 海台与海山
洋中脊和转换断层的岩浆与构造活动在板块边 界上产生大量海山 ${ }^{[1]}$. 与之对应, 地幔热点则主要表 现为板块内部的火山活动 ${ }^{[2]}$. 当洋中脊和热点这两个 岩浆单元相互作用时, 热点的活动强度会得到放大, 形成范围宽广的海台 ${ }^{[3]}$. 这些海台、海山(或者海山 链) 忠实地反映了岩浆的活动强度和热点的运动轨 迹 $^{[2,4 \sim 7]}$, 因此可以根据热点成因海山的形成年代、位 置和岩浆熔融总量来讨论热点活动强度的时变性、周 期性与热点-洋中脊-转换断层交互作用的关系 ${ }^{[4,5,8,9]}$. 岩石的放射性测年可以直接测定海山的形成年代,
而海洋地壳厚度(层 $2 \mathrm{~A}$ 至层 3 ) 可以近似代替地幔岩 浆熔融总量 ${ }^{[10]}$. 但是到目前为止, 海底岩石采样标本 和海底地震速度剖面确定的地壳厚度资料都非常稀 少. 在一些研究较少的区域, 无法利用上述方法推算 热点的轨迹及岩浆通量. 其他地球物理方法, 如地形 或重力数据推算的地壳厚度、磁条带推算的地壳年龄 以及在此基础上的板块重构等，也就成为认识此类 问题的主要手段 ${ }^{[6,11]}$.

与东太平洋隆起和大西洋中脊相比, 西南印度 洋区域在历史上的研究程度较低. 近年来, 西南印度

英文引用格式: Zhang T, Lin J, Gao J Y. Interactions between hotspots and the Southwest Indian Ridge during the last 90 Ma: Implications on the formation of oceanic plateaus and intra-plate seamounts. Sci China Earth Sci, 2011, doi: 10.1007/s11430-011-4219-9 
洋中脊超慢速扩张和斜向扩张的特征逐渐引起了国 际上的广泛关注 ${ }^{[12]} .2007$ 年, 中国在西南印度洋中脊 上发现了第一个活动的热液硫化物喷 ${ }^{[13]}$. 科学与 资源的双重意义使得西南印度洋成为目前国际深海 研究的焦点区域. 在本文的研究区域(图 1)内, 除北 东向横贯的西南印度洋中脊以及马里昂(Marion)、克 洛泽(Crozet)、康拉德(Conrad, 已经不再活动)等热点 外, 还包括马达加斯加(Madagascar)海台、德尔卡诺 (Del Cano) 隆起、康拉德隆起等海台以及众多未命名 的海山. 这些海台和海山的形成很早就被认为与洋 中脊、热点有着密切的联系 ${ }^{[14 ~ 16]}$. 由于缺少定量的大 尺度岩浆通量数据和海台(海山)测年资料, 我们对马 里昂、克洛泽热点活动强度的时变性, 德尔卡诺隆 起、马达加斯加海台的起源, 以及热点与西南印度洋

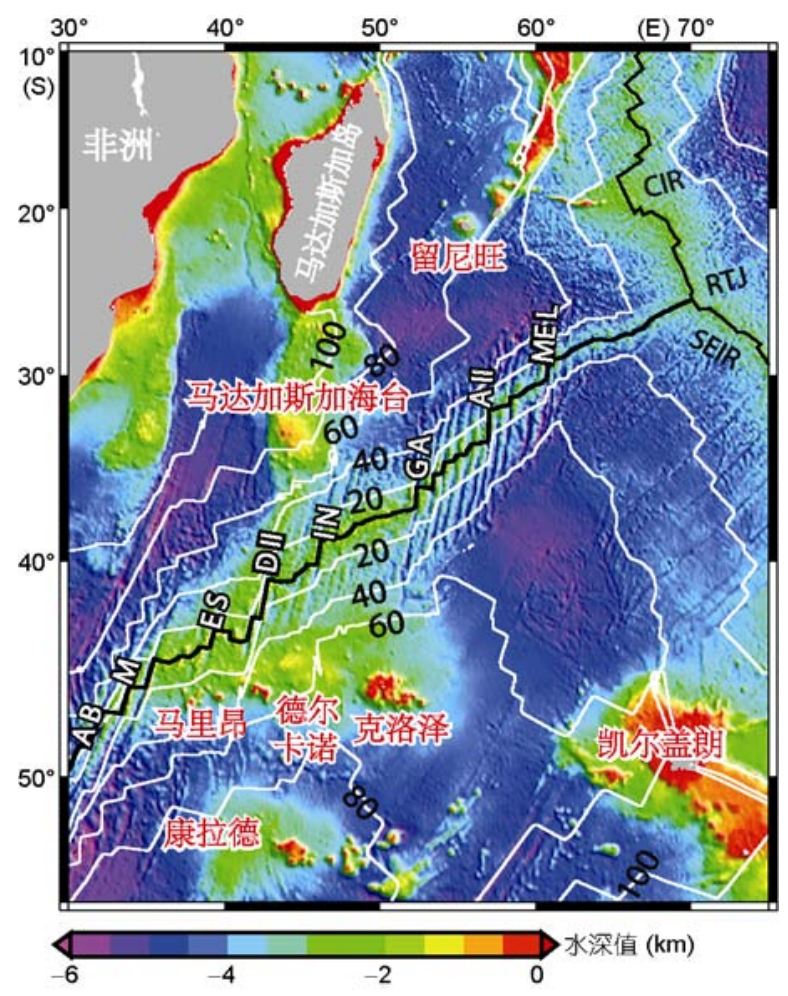

图 1 基于卫星测高数据的研究区域水深 ${ }^{17]}$

热点及海台用红色字体表示, 主要转换断层用白色字体表示, 其中 $\mathrm{AB}$ 表示安德鲁本(Andrew Bain), M 表示马里昂(Marion), ES 表示埃 里克辛普森(Eric Simpson), DII 表示探索 II (Discovery II), IN 表示因 道姆(Indomed), GA 表示加里艾尼(Gallieni), AII 表示亚特兰提斯 II (Atlantis II), MEL 表示梅尔维尔(Melville). 地壳年龄用间隔为 20 $\mathrm{Ma}$ 的白色等值线表示 ${ }^{[18]}$, 并且用黑色数字标注. 黑线表示活动扩 张中心位置, CIR 为中印度洋中脊, SEIR 为东南印度洋中脊, RTJ 为 罗德里格斯三联点
中脊交互作用的关系仍然没有清晰的认识.

本文先结合板块重构确定了西南印度洋区域内 热点-中脊相对位置和海台、海山的形成年代. 然后通 过水深异常和艾里均衡模式计算了相应的热点熔融 总量, 重点研究以下问题: (1) 分析研究区域内的海 台和海山的起源与演化过程及其与热点-洋中脊的关 系; (2) 追踪热点的运动轨迹, 分析热点活动强度在 时间和空间上的变化; (3) 讨论热点-洋中脊-转换断 层的相互作用及其对中脊几何形态产生的影响.

\section{1 地质背景}

横贯研究区域的西南印度洋中脊与众多的转换 断层构成了西南印度洋最为显著的地形特征. 西南 印度洋中脊最早形成于 $150 \mathrm{Ma}$ 以前 ${ }^{[19]}$, 并从 $80 \mathrm{Ma}$ 开始快速延伸变长. 从 $80 \mathrm{Ma}$ 到现在, 洋中脊由 DII 转换断层向现在的罗德里格斯三联点 $\left(\mathrm{RTJ}, 70^{\circ} \mathrm{E}\right)$ 方 向延伸了 $2500 \mathrm{~km}$, 逐步形成了 GA, AII 和 MEL 等转 换断层 ${ }^{[19]}$. 目前, 长约 $1000 \mathrm{~km}$ 的 $\mathrm{AB}$ 转换断层将西 南印度洋分成东西两部分 ${ }^{[19]}$. 在 $A B$ 转换断层以东直 至 RTJ, 前人的地球物理和地球化学研究都表明岩浆 通量有由西向东减少的趋势 ${ }^{[20 ~ 22]}$. 根据洋中脊的地 球物理(水深、侧扫、重力、磁力)、几何形状(中脊段 长度、斜向扩张角度、转换断层出现频率) 和地球化 学、岩石学的特征, $\operatorname{DII}\left(42^{\circ} \mathrm{E}\right) 、 \mathrm{GA}\left(52^{\circ} 20^{\prime} \mathrm{E}\right)$ 和 $\operatorname{MEL}\left(60^{\circ} 45^{\prime} \mathrm{E}\right)$ 这三个转换断层将西南印度洋中脊东 段分为 4 个超级段 ${ }^{[23,24]}$. AB 到 DII 区域水深最浅, 对 应于负的剩余地幔布格重力异常(RMBA), 显示此处 存在强烈岩浆活动导致的增厚地壳或者较高的地幔 温度; 此区可能受到了马里昂热点的影响 ${ }^{[23 \sim 25]}$. 由 DII 向东至 $49^{\circ} 30^{\prime} \mathrm{E}$, 海底变深、RMBA 变高, Georgen 等 ${ }^{[25]}$ 据此推断马里昂热点的向东传播被 DII 转换断 层所阻断. 自 GA 到 MEL 区域地震速度剖面确定的 地壳厚度明显偏薄 ${ }^{[26]}$. 由 MEL 到 RTJ 是平均水深最 深的区域, 地震速度剖面显示地壳厚度继续减薄(平 均 4 5 km 厚) ${ }^{[27,28]}$, 在此区域缺少转换断层, 出露大 量的蛇纹岩化的橄榄岩, 具有典型的超慢速扩张中 脊特征.

在中脊北侧的非洲板块上，水深最浅的区域包 括莫桑比克脊、马达加斯加海台以及马达加斯加海台 延伸到扩张中脊的脊状地形. 马达加斯加海台的地 壳性质还存在争议, Coffin 等 ${ }^{[15]}$ 根据 ODP 第 25 航次 
246 和 247 点的钻探资料将之划为陆壳, 但是这两个 钻孔都没有钻透沉积物. 地震速度剖面数据显示虽 然马达加斯加海台南北两个部分 (以 $31^{\circ} \mathrm{S}$ 为界)的地 壳结构有所不同, 但是都更倾向于洋壳, 并且可能源 于白严纪的热点活动 ${ }^{[29]}$. 马达加斯加海台延伸到扩 张中脊的脊状隆起被认为是马里昂热点的活动轨 迹 ${ }^{[25,30]}$. 而在中脊南面的南极洲板块上面, 存在着康 拉德隆起、马里昂热点、德尔卡诺隆起以及克洛泽海 台等众多的海底高地. 以西南印度洋中脊为中心, 与 马达加斯加海台共轭伴生的康拉德隆起的最浅处已 经接近海平面, 其东南侧的海山 $\left(48^{\circ} \mathrm{E}, 53^{\circ} \mathrm{S}\right)$ 下可能 存在一个已经不再活动的热点 ${ }^{[31]}$. 马里昂热点、德尔 卡诺隆起以及克洛泽海台一起构成了一个直径超过 $1000 \mathrm{~km}$ 的热点隆起, 有些学者将它们归为一个热点 群. 马里昂热点是少数几个现今仍然活动的古老热 点之一, 其 $88 \mathrm{Ma}$ 时的岩浆作用生成了马达加斯加岛 南部的 Volcan de l'Androy 熔岩区, 可能造成了印度 与马达加斯加岛的大陆分裂 ${ }^{[32]}$. Coffin 等 ${ }^{[15]}$ 推断德尔 卡诺隆起可能源于马里昂热点的岩浆活动. Goslin 等 ${ }^{[33]}$ 通过对德尔卡诺隆起的水深-重力导纳函数的分 析, 认为此处接近局部均衡状态, 可能产生于岩石圈 较弱的洋中脊. 德尔卡诺隆起和克洛泽海台位置相 近, 但是均衡分析及地震的速度剖面都说明它们的 成因不同 ${ }^{[34]}$. 克洛泽海台现在仍然活跃, 但是其起源 及轨迹仍然存在着很大的争议 ${ }^{[31,35,36]}$.

\section{2 西南印度洋的剩余水深}

水深数据是热点、洋中脊岩浆活动的直观表现形 式. 由于海山及海台的地形受到岩石圈沉降、沉积物 充填、压实等后期作用的改造, 因此本文从观测水深 中逐一去除这些影响因素, 得到反映地壳生成初期 岩浆活动状态的剩余水深.

\section{1 数据来源及误差}

本文使用的水深数据为 Smith 和 Sandwell 最新 版本(11.1)的 $1^{\prime} \times 1^{\prime}$ 卫星测高反演数据 ${ }^{[17]}$, 如图 1 所示. 在融合了更多船测资料后，此数据 $75 \%$ 的区域在 24 $\mathrm{km}$ 的范围内都有船测数据的控制. 在地形复杂的区 域, 此数据精度比之前版本有了大幅的提高, 因此非 常适合用来研究洋中脊、海山这些水深变化较大的区 域. 沿西南印度洋中脊, 本文利用 $100 \mathrm{~m} \times 100 \mathrm{~m}$ 网格
间距的船测多波束数据(源于法国 L'Atalante 号 Gallieni 航次 $\left.{ }^{[20]}\right)$ 与卫星测高 11.1 版本的水深数据进 行了对比, 并采用 Smith 和 Sandwell ${ }^{[17]}$ 的评价方法进 行精度评价. 多波束覆盖区域的水深变化的均方根 为 $4613 \mathrm{~m}$, 而卫星测高数据与多波束数据的差值的 均方差仅为 $198 \mathrm{~m}$, 意味着最新版本的卫星测高反演 数据置信度可以达到 $96 \%$.

地壳年龄数据采用 Muller 最新发布的 $2^{\prime} \times 2^{\prime}$ 的海 洋地壳年龄数据模型, 如图 2 所示. 在研究区域内 ${ }^{[18]}$, 相比之前广泛应用的 $6^{\prime} \times 6^{\prime}$ 地壳年龄模型, $2^{\prime} \times 2^{\prime}$ 模型 加入了更多的船测地磁数据. 在整个研究区域内, 其 $58.9 \%$ 区域的地壳年龄数据误差小于 $2 \mathrm{Ma}, 93.7 \%$ 区 域的误差小于 $5 \mathrm{Ma}$. 数据误差大于 $5 \mathrm{Ma}$ 的区域多 为洋陆交界处或转换断层破裂带, 对我们研究内容 的影响不大. 现今马里昂和克洛泽热点所处的地壳 年龄分别为 30 和 $70 \mathrm{Ma}$ 左右, 康拉德隆起的地壳年 龄约为 $90 \mathrm{Ma}$.

本文使用的沉积物厚度为 Divins 提供的 $5^{\prime} \times 5^{\prime}$ 的网格化数据 ${ }^{[37]}$, 如图 3 所示. 此数据来源于已发表 的沉积物等厚图、DSDP 和 ODP 的钻井资料以及 NGDC, IOC 和 GAPA 项目. 研究区域内的平均沉积 物厚度为 $0.75 \mathrm{~km}$, 除靠近陆地的区域外, 沉积物厚 度基本与地壳年龄正相关，并且明显在海台和热点 处较厚, 其中马里昂和克洛泽热点区域厚度均接近 $1 \mathrm{~km}$.

\section{2 计算方法}

岩石圈在向两侧扩张的过程中不断变冷、变重, 进而导致岩石圈和海底的不断沉降. 因此无论基于 半空间模型还是基于板块冷却模型的岩石圈沉降公 式都将地壳年龄作为主要控制因素 ${ }^{[38 \sim 40]}$. 相比其他 方法, Stein 和 Stein ${ }^{[40]}$ 根据热流数据与水深数据的相 关性提出的改正公式(1)简单易用, 并且在年龄较老 的地壳上理论值与观测值更加一致. 因此本文采用 这个公式来计算剩余水深.

$$
\begin{gathered}
D=2600+365 t^{1 / 2}, \quad t<20 \mathrm{Ma}, \\
D=5651-2473 \exp (-0.0278 t), t \geqslant 20 \mathrm{Ma},
\end{gathered}
$$

其中 $D$ 为正常沉降值 $(\mathrm{m}), t$ 为地壳年龄 $(\mathrm{Ma})$.

沉积物对水深数据的影响有两方面，一方面沉 积物的填充与压实使得水深变浅，另一方面，沉积物 的负载会使得岩石圈发生均衡的调整，导致沉积基 底变深. 在将沉积物厚度从观测水深中去除后，经验 


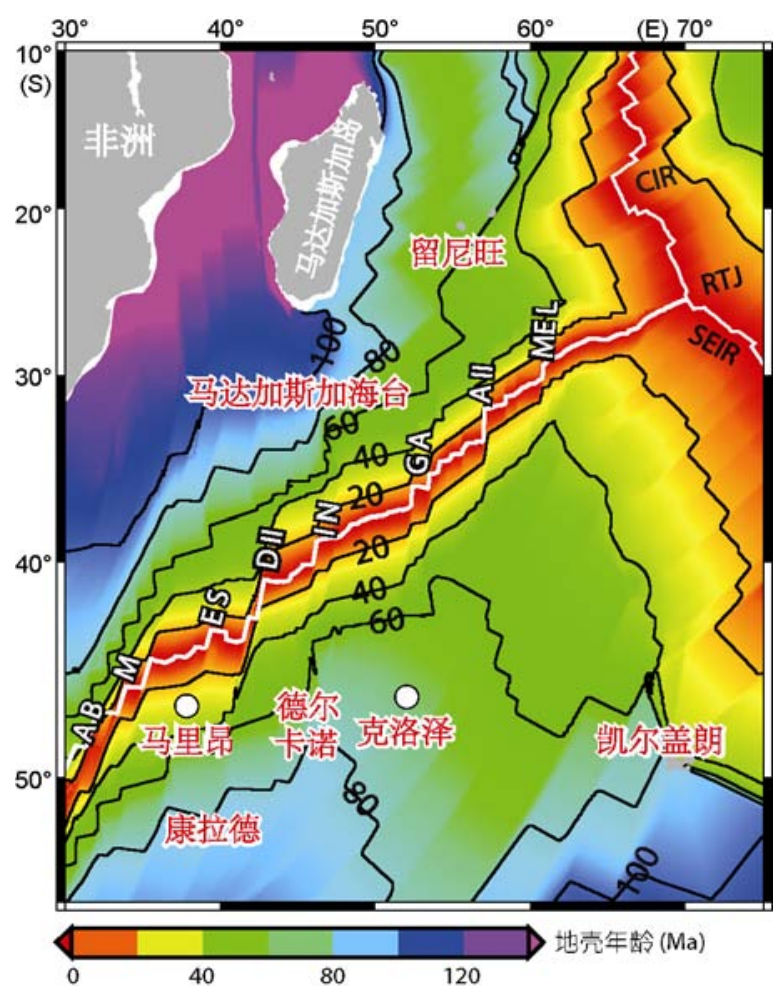

图 2 等值线间隔为 $20 \mathrm{Ma}$ 的海洋地壳年龄模型 ${ }^{[18}$ 地壳年龄用黑色数字标注, 热点位置用白色实心圆表示, 热点及海 台名称用红色字体表示, 主要转换断层用白色字体表示

公式(2)被用于计算沉积物负载造成的岩石圈变形. 公式(2)是对 $\mathrm{Crough}^{[41]}$ 利用 DSDP 资料计算的沉积物 改正值的简单拟合 (原始值与拟合值的均方差约为 $0.06 \mathrm{~km})$ ，适用于沉积物厚度小于 $1.8 \mathrm{~km}$ 的区域:

$$
S=0.22 c+0.00014 c^{2}-c,
$$

其中, $S$ 为沉积物效应改正值 $(\mathrm{m}), c$ 为沉积物厚度 $(\mathrm{m})$. 因此剩余水深即为

$$
R B=B-D+S,
$$

其中, $R B$ 为剩余水深, $B$ 为观测水深值.

\section{3 剩余水深异常}

在剩余水深图中(图 4), 以马里昂热点、德尔卡 诺隆起和克洛泽热点为主体的巨大隆起是最为明显 的异常特征区域. 和世界上其他大多数的热点一样, 这种隆起源于地幔热物质上涌形成的支撑力以及热 点的岩浆熔融作用, 其范围大约为 $1000 \mathrm{~km}$. 以马里 昂-德尔卡诺-克洛泽为中心, 向南及向东方向的隆起 范围不超过 $300 \mathrm{~km}$ ，而在靠近洋中脊的北、西方向， 其范围却接近 $1000 \mathrm{~km}$, 可能是马里昂、克洛泽热点

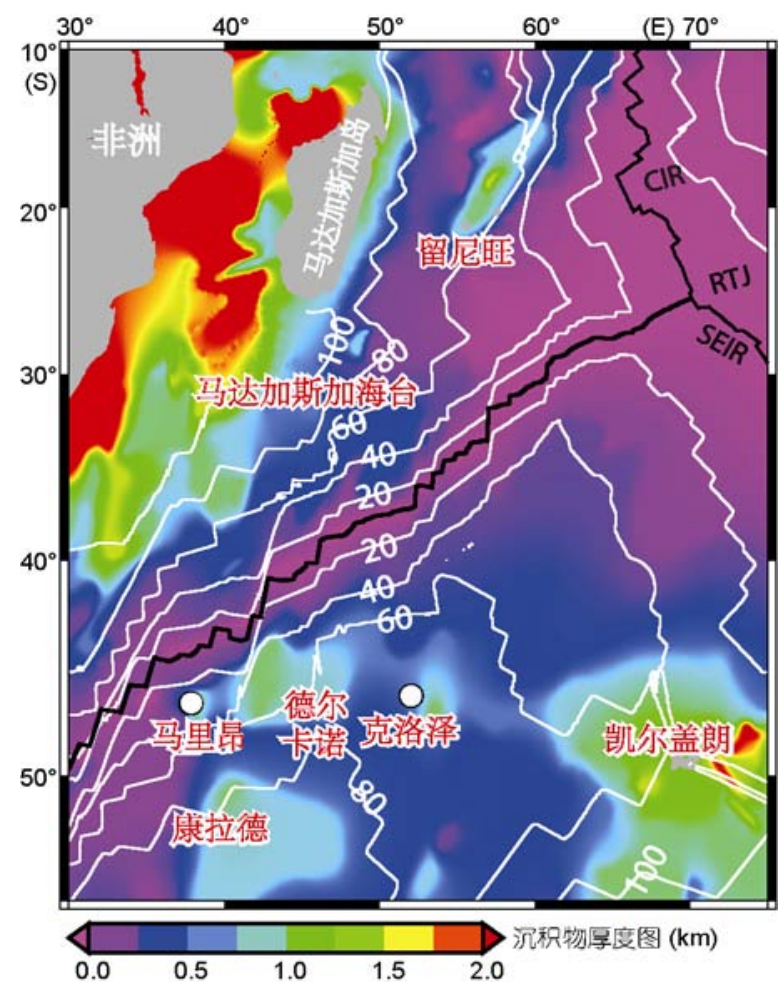

图 3 沉积物厚度 ${ }^{[37]}$

热点位置用白色实心圆表示, 热点及海台名称用白色字体表示, 间 隔为 $20 \mathrm{Ma}$ 的地壳年龄等值线用白色数字表示

的作用受到了中脊放大的结果.马达加斯加海台的 南端和康拉德隆起以洋中脊为中心对称分布，具有 相近的剩余水深值. 马达加斯加海台延伸到西南印 度洋中脊的脊状地形被认为是热点的轨迹 ${ }^{25,30]}$. 沿 三联点轨迹, IN 到 AII 转换断层之间存在一个南北对 称的地形隆起区域，此区域总体上呈现由西向东逐 步收缩的“V”字形状. 在三联点轨迹以内, GA 转换断 层以西的水深值明显小于 GA 以东. 这不仅说明 GA 转换断层以西的中脊段可能受到了热点的影响 ${ }^{[25]}$, 而且表明自 GA 转换断层形成开始, 其西侧的中脊段 就一直受到热点的影响. IN 和 GA 转换断层之间活动 扩张脊处地形异常也表现为由西向东收缩的“V”字形 状，可能受到了克洛泽热点的影响 ${ }^{[42]}$. 与慢速扩张的 大西洋中脊类似, GA 至 AII 转换断层之间频繁发育 的转换断层内角表现出明显的高地形. 由于在慢速超慢速扩张中脊上，中脊段被认为是两个拆离断层 间应力转换的区域，因此这种高地形可能是源于拆 离断层的下盘上升的作用 ${ }^{[43]}$, 而非岩浆活动的结果. 


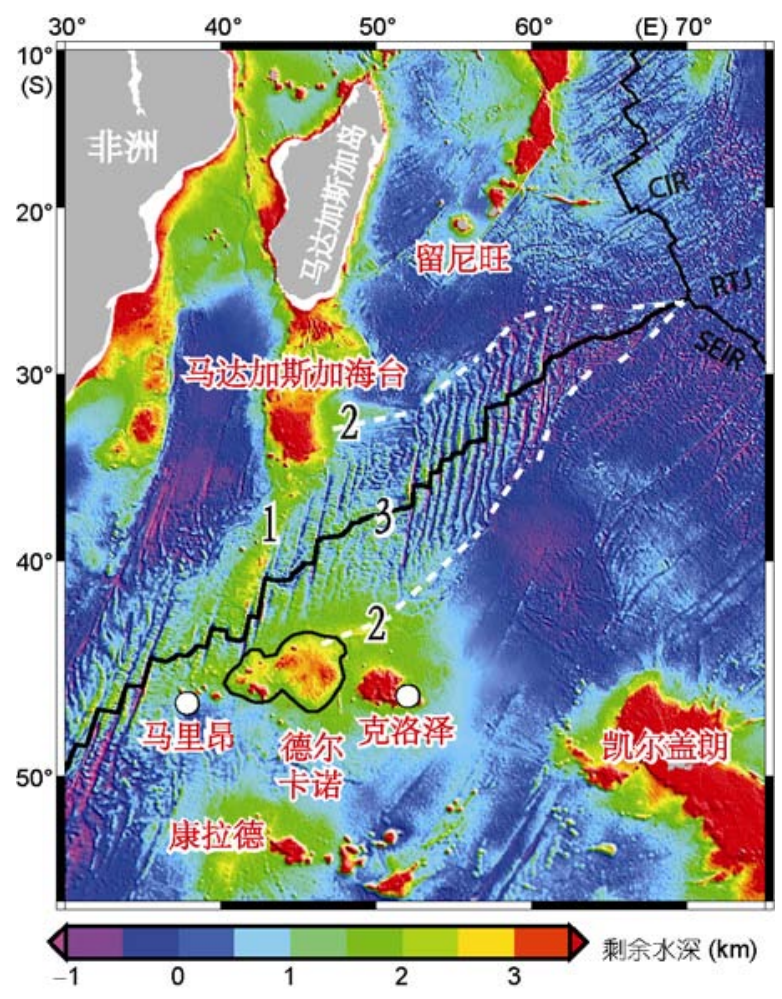

图 4 计算的西南印度洋剩余水深

热点位置用白色实心圆表示, 热点及海台名称用红色字体表示, 活 动扩张中心用黑线表示, 三联点轨迹用白色虚线表示, 德尔卡诺隆 起的位置用黑线圈出, 黑色数字表示本文讨论的未命名地形异常区, 其中: 1 为马达加斯加海台延伸到西南印度洋中脊的脊状地形, 2 为 沿三联点轨迹自 IN 到 AII 转换断层的对称“V”字形地形, 3 为 IN 到 GA 转换断层间的地形隆起

\section{3 板块的重构}

本文使用 Muller 等 ${ }^{[31}$ 基于大西洋-印度洋热点参 考系的有限欧拉极进行板块重构(图 5). 研究区域内 包括非洲、南极洲、澳大利亚和印度-中印度海盆四 个主要板块, 板块边界的数据来源于 Nuvel-1 模型 ${ }^{[44]}$. 由于地球化学和岩石学数据明确证明马里昂热点在 $90 \mathrm{Ma}$ 左右时位于马达加斯加岛上 ${ }^{[32]}$, 之后才慢慢与 扩张中脊相接近, 因此我们重构的开始时间选为 $90 \mathrm{Ma}$

如图 5(a)所示, 马里昂热点在 $90 \mathrm{Ma}$ 时位于马达 加斯加岛的东部, 即现在的 Volcan de l'Androy 熔岩 区位置, 与此前的地球化学证据一致 ${ }^{[32]}$. 此时, 具有 相近剩余水深的马达加斯加海台和康拉德海台在位 置上重合, 而三联点位于马达加斯加海台东侧, 并且 缓慢地向北东方向移动. 在 $84 \mathrm{Ma}$ 时(图 5(b)), 马里
昂位于马达加斯加海台的北部, 此时康拉德和马达 加斯加海台基本分开, 康拉德隆起所在的地壳已经 形成. 之后, 随着非洲板块和三联点的北东向移动, 古 RTJ 逐步靠近马里昂热点(图 5(c)). 在 $73.6 \mathrm{Ma}$ (图 $5(\mathrm{~d})$ ) 时, 古 RTJ 距离马里昂热点只有 $160 \mathrm{~km}$, 而克洛 泽热点位于印度大陆的南东侧, 现在克洛泽热点所 处地壳的南部已经出现. 自 73.6 68.5 Ma(图 5(d), 5(e)), 古 RTJ 进一步接近马里昂热点, 在中脊的两侧 分别形成德尔卡诺隆起的东部和马达加斯加海台的 东南部. 自 68.5 58.5 Ma(图 5(e), 5(f)), 古 RTJ 快速 向北东方向移动, 连续而稳定地生成了 $\mathrm{IN}, \mathrm{GA}$ 和 $\mathrm{AII}$ 转换断层, 其移动的速度达到了现今扩张速率的 8 倍. 此时马里昂热点接近或者位于洋中脊扩张中心, 强 烈的岩浆活动导致德尔卡诺隆起的中部开始形成. 热点沿中脊热效应的传播在 IN-GA-AII 转换断层间 中脊段上形成了由西向东收缩的长度超过 $1000 \mathrm{~km}$ 的“V”字形海台. 海台随着中脊的扩张分裂为现在沿 三联点轨迹南北对称的地形突起(现在的位置见图 4). 克洛泽热点此时也经过东南印度洋中脊进入了南极 洲板块, 但是在其轨迹上没有明显的地形异常. 50.3 $\mathrm{Ma}$ 时(图 5(g)), 马里昂热点接近或者位于 DII 到 IN 转换断层之间的洋中脊上, 在中脊两侧形成了对称 的地形高异常, 其北侧为马达加斯加海台的南末端, 而在南侧为德尔卡诺隆起的中部区域. 42.7 35.5 $\mathrm{Ma}$ (图 5(h), 5(i)) 时, 扩张中心逐渐远离马里昂热点, 马里昂热点位于 AII 转换断层的东侧年龄为 $53 \mathrm{Ma}$ 的 地壳上, 形成德尔卡诺隆起的西部区域. 而此时转换 断层西侧开始形成马达加斯加海台到洋中脊的脊状 突起. 由于其对称的中脊南侧没有对应地形异常, 因 此推断此地形异常不是形成于洋中脊. $20.5 \mathrm{Ma}$ 到现在 (图 5(j) 5(1)), 非洲板块相对马里昂热点有一个明显的 变向, 由原来的近正北向改为东向运动, 马里昂到达 当前热点活动位置. 相比活跃的马里昂热点, 在整个 过程中, 克洛泽热点轨迹并不存在明显的地形异常.

\section{4 岩浆熔融通量的估算}

相比其他方法，使用水深数据计算岩浆通量更 加直观、便捷，因此得到广泛的应用，如前人对太平 洋的夏威夷热点以及大西洋的 Walvis 和 St. Helena 热点的岩浆通量研究 ${ }^{[8,9,45]}$. 若将莫霍面视为补偿面, 在已知剩余水深后，根据岩浆区域的均衡补偿模式， 


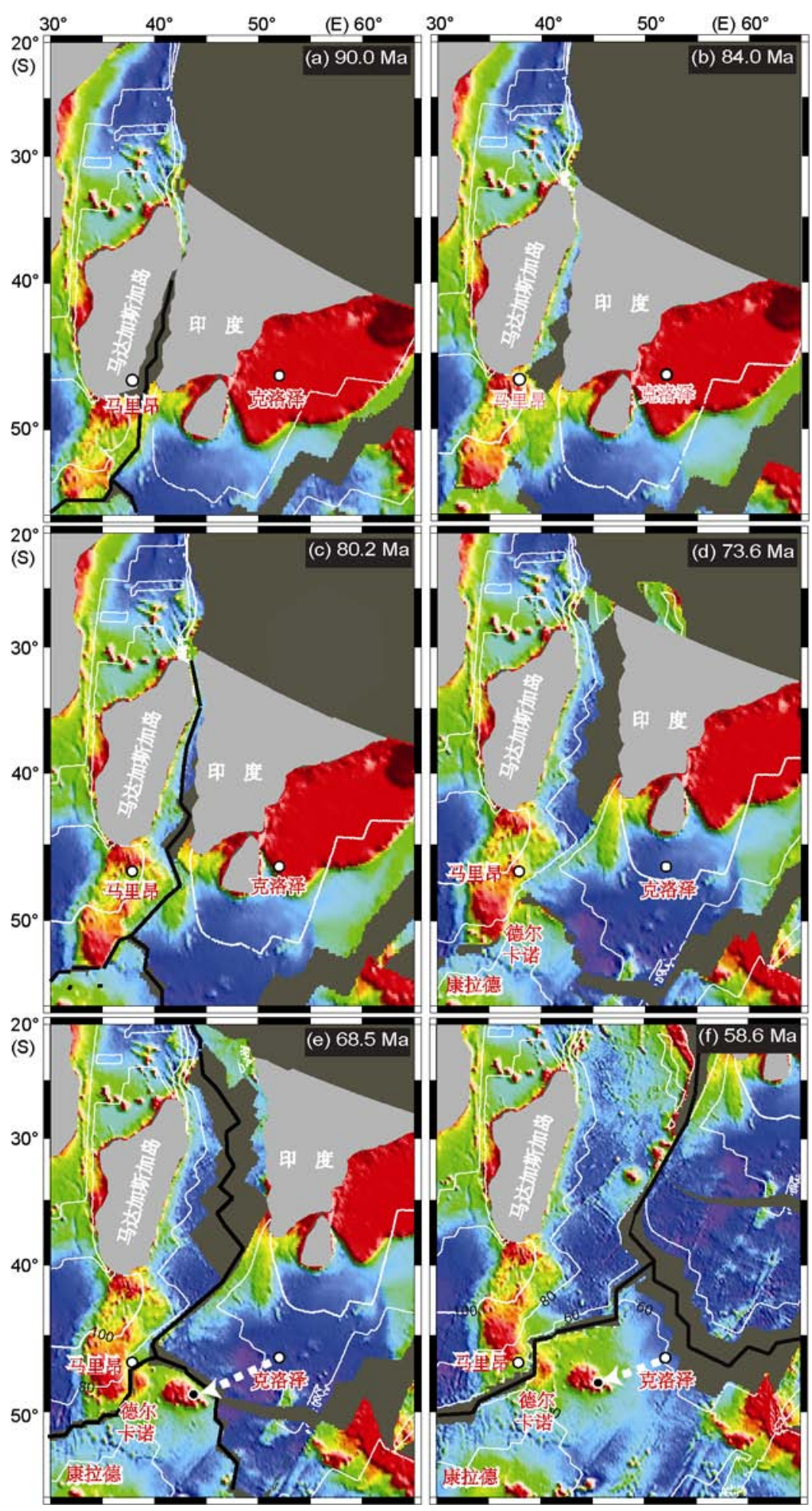




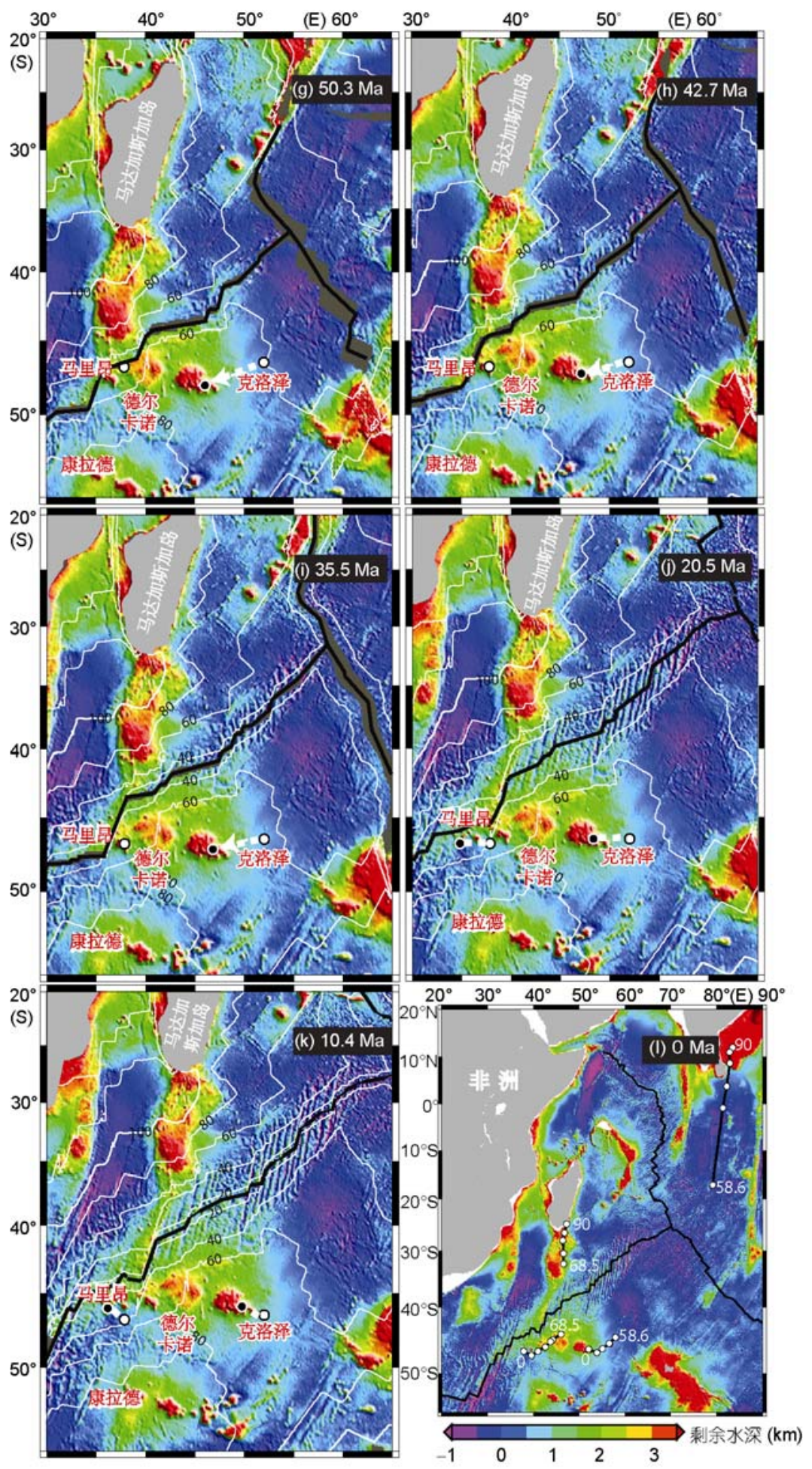

图 5 基于热点绝对坐标系的西南印度洋板块重构

(a) (k), 底图为相应年代的剩余水深图, 间隔为 $20 \mathrm{Ma}$ 的地壳年龄用白线表示, 并用黑色数字标注, 古洋中脊位置使用黑线表示, 马里昂热 点和克洛泽热点的位置用白色实心圆表示, 热点现在所在位置用黑色实心圆表示, 两者用白色虚线连接; (1), 热点在相应板块上运动的轨迹, 白色实心圆对应不同年龄的热点位置, 起止年龄用白色数字表示, 热点轨迹用黑色线表示 
就可以推算出补偿面的形状, 进而计算地壳厚度. 由 于洋中脊的岩石圈非常薄弱, 在此区域产生的海山 多接近局部均衡模式. 因此, 本文根据艾里(Airy)局 部均衡模式计算洋中脊处生成的地壳厚度:

$$
F_{\text {Airy }}=\left(1+\frac{\rho_{\mathrm{v}}-\rho_{\mathrm{w}}}{\rho_{\mathrm{m}}-\rho_{\mathrm{v}}}\right) h+R,
$$

其中 $\rho_{\mathrm{v}}$ 为火山地壳的密度, $\rho_{\mathrm{w}}$ 为海水密度, $\rho_{\mathrm{m}}$ 为地幔 密度, 这里分别取 2800,1030 和 $3300 \mathrm{~kg} \mathrm{~m}^{-3} ; R$ 为平 均地壳厚度, 这里取 $6 \mathrm{~km}$, 使得德尔卡诺隆起区域 的计算地壳厚度与地震速度剖面确定地壳厚度相符 合 ${ }^{[34]}$. 计算得到的地壳厚度如图 6 所示.

根据板块重构提供的海山生成年代, 本文对相 应年龄段的地壳厚度进行积分, 得到马里昂热点岩 浆通量随时间的变化. 为了去除正常地壳和热点隆 起的作用(详见 5.1 节), 本文将 $6 \mathrm{~km}$ 的地壳厚度从熔 融异常部分中去除. 在 80.2 42.7 Ma(图 5(c) (h)) 期 间, 马里昂热点与三联点、中脊非常接近, 其岩浆强 度可以通过地壳的连续增生过程来表示. 从 $42.7 \mathrm{Ma}$

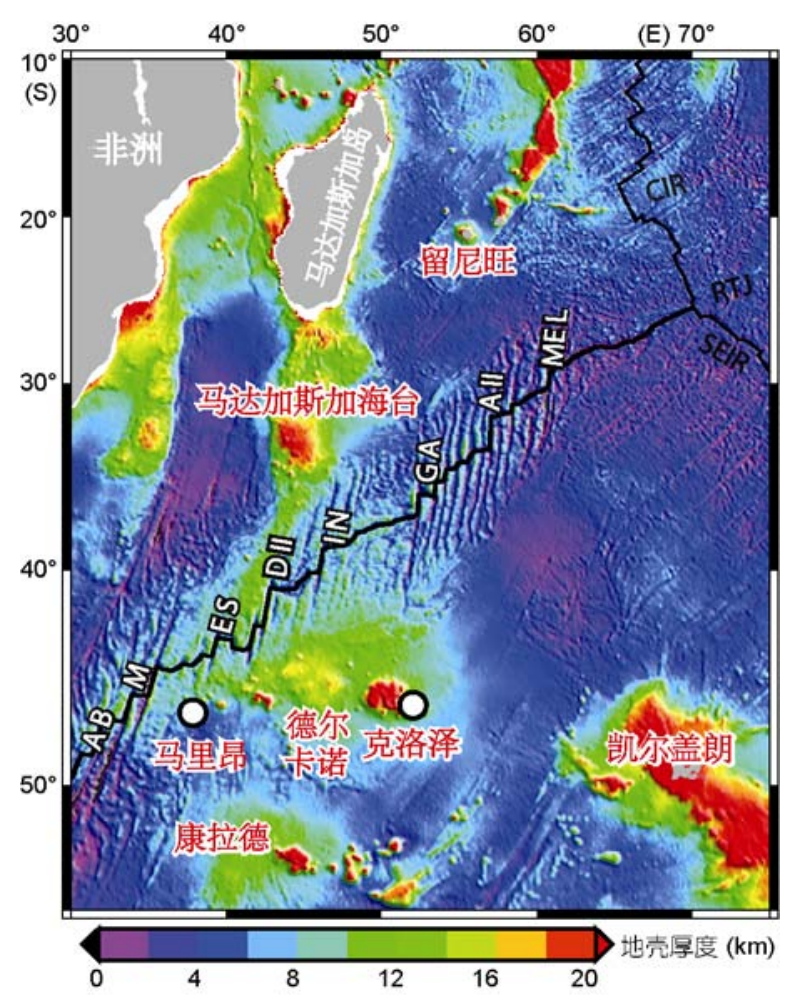

图 6 基于艾里均衡模式计算的地壳厚度

热点位置用白色实心圆表示, 热点及海台名称用红色字体表示, 主 要转换断层用白色字体表示, 活动扩张中心用黑线表示
到现在(图 5(h) (1)), 热点的主要表现形式为板内火 山, 不是一个连续的过程, 因此本文采用时间段上的 平均值来表示岩浆通量大小. 由图 7(a)可以看出, 在 中脊靠近热点时候, 热点的作用得到了放大, 形成了 连续的强岩浆活动. 74 和 $50 \mathrm{Ma}$ 左右的两个剩余岩浆 通量的高峰期正对应于马里昂热点靠近古 RTJ 和位 于洋中脊上的两个时期, 分别形成了现在德尔卡诺 隆起的东部和中部主体. 在 42.7 35.5 Ma 之间, 马里 昂热点的作用多表现为板内火山, 其平均岩浆通量 明显小于热点和中脊发生作用的阶段, 最高平均值 仅为 74 和 $50 \mathrm{Ma}$ 时的 1/6 1/5(图 7(a)). 之后, 随着热 点远离洋中脊, 其剩余岩浆通量进一步降低. 马里昂 热点的这种活动强度随时间减小的趋势与 Georgen 等的推断相同 ${ }^{[25]}$. 而热点轨迹上的剩余水深值(图 7(b))表明, 在热点远离中脊阶段某些时刻(约 $30 \mathrm{Ma}$ ) 的水深却比 74 和 $50 \mathrm{Ma}$ 时的水深还要浅, 说明热点 在板内的岩浆活动更加集中, 容易形成高地形的海 山, 而在与洋中脊相互作用时, 沿中脊传播的热点作 用则容易导致广阔的低幅值海台.

\section{5 讨论}

\section{1 模型误差及其对结果的影响}

本文假定研究区域内的海台和海山符合艾里均 衡模式. 热点在板内活动时, 岩石圈已经具有一定的 强度, 因此在计算补偿面时要考虑海山生成时的岩 石圈有效弹性厚度，使用区域补偿而非局部补偿模 型计算岩浆熔融总量. 但是针对夏威夷海山的研究 表明, 不同的补偿模式只改变补偿面的形状, 而不会 影响熔融总量的幅值 ${ }^{[8]}$. 由于本文的研究重点是岩浆 通量, 因此采用艾里模式不会对结果产生明显影响.

在热点活动区域, 范围达到数千公里的热点隆 起可能源于岩浆的熔融作用, 也有可能源于地幔支 撑力. 如图 8 所示, 前一种模型在计算岩浆通量中要 包括热点隆起的部分, 后者则需要扣除热点隆起的 影响. 由于针对夏威夷海山的研究认为这种隆起源 于地幔支撑力 ${ }^{[8]}$, 因此本文在计算岩浆通量时去除了 隆起部分的影响. Wessel ${ }^{[46]}$ 系统总结了各种消除热点 隆起作用的优缺点, 这里我们利用简单的洋中脊地 形的对称性来去除这种影响. 考虑到沿三联点轨迹 的 IN-AII 转换断层间的对称地形源于热点作用沿洋 

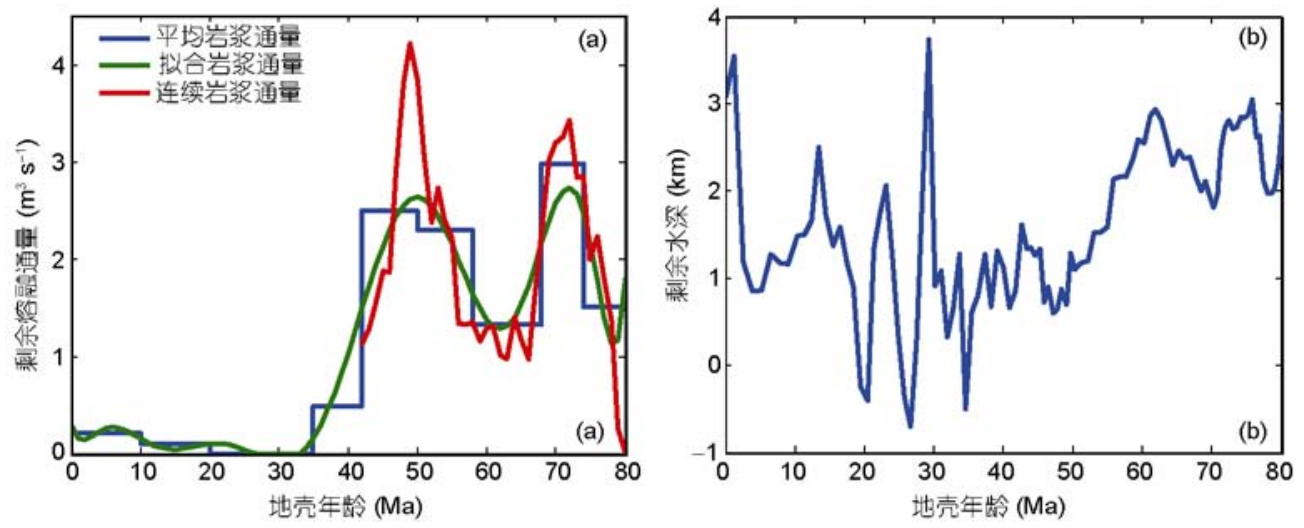

图 7 计算的马里昂热点活动强度随时间的变化

(a) 马里昂热点剩余岩浆熔融通量随时间变化; (b) 马里昂热点轨迹上剩余水深随时间变化

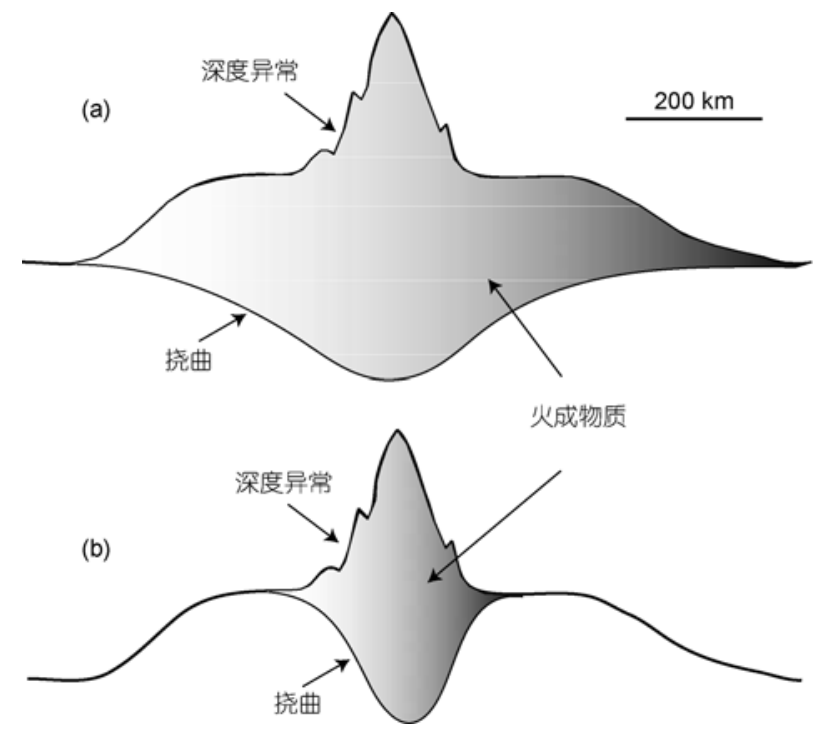

图 8 热点隆起模式示意

修改自 Vidal 等 ${ }^{[8]}$. (a) 岩浆通量包含热点的隆起; (b) 岩浆通量不包 含热点的隆起

中脊传播生成的同一个海台, 两者应该具有一致的 水深值. 由于北侧地形并没有受到热点隆起的影响, 所以南侧与北侧水深值的差值即为隆起的校正值. 另外, 由于热点隆起中心区域面积巨大且幅值变化 较小, 所以我们采用了统一的校正值.

文中所用的板块重构数据是基于大西洋-印度洋 热点绝对静止参考系, 而现在越来越多的证据证明, 热点本身并不是固定不动的 ${ }^{[4]}$. 但是热点的运动速 度相对板块运动的速度较慢, 在西南印度洋的热点 多又具有相似的运动轨迹 ${ }^{[47]}$. 热点的运动在本文讨 论的大尺度问题上基本不影响讨论的结果.

\section{2 马达加斯加海台以南的脊状地形}

前人认为, 马达加斯加海台延伸到西南印度洋 中脊的脊状地形是马里昂热点的轨迹 ${ }^{[25,30]}$, 热点沿 此路径穿过西南印度洋洋中脊的 ES-DII 段, 到达现 在位置. 本文重构显示热点轨迹多位于在 DII 的东侧, 位置上与此脊状地形并不对应, 而且也不存在热点 穿过 ES-DII 段中脊时形成的海台. 因此, 本文认为 此地形隆起的形成可能是位于 DII 东侧的马里昂热 点透过转换断层的作用结果. 这种模式与南大西洋 的 Discovery 热点透过厄加勒斯(Agulhas)转换断层在 另一侧形成的海山链相似. 即由于转换断层两侧的 年龄不一致, 转换断层两侧的岩石圈底界面会存在 一定的坡度, 从而使得热点的作用从转换断层的一 侧传播到另一侧, 导致在另一侧形成链状海山 ${ }^{[48]}$.

\section{3 德尔卡诺隆起的形成}

德尔卡诺隆起及其周围地形异常几乎包括了热 点的所有活动形式: 与三联点、洋中脊、转换断层的 交互作用以及板内海山活动. 80 68.5 Ma 时古 RTJ 与 热点的作用形成德尔卡诺隆起的东半部分, 之后持 续与洋中脊的共同作用形成了现在德尔卡诺隆起的 当中主体部分, 而其西部的海山是由于 $42.5 \sim 35 \mathrm{Ma}$ 之间的板内海山形成. 由于三联点与洋中脊部分岩 石圈较弱, 更容易接近艾里局部均衡状态, 因此德尔 卡诺隆起主体部分处于局部均衡的状态, 而板内的 海山则主要处于区域均衡状态. 这与 Goslin 等 ${ }^{[29]}$ 利 用重力数据对德尔卡诺和克洛泽均衡状态的解释一 致. 


\section{4 马里昂热点熔融异常的时变性}

本文将马里昂热点的剩余熔融通量和夏威夷及 冰岛热点的剩余熔融通量进行了比较 ${ }^{[4,5]}$, 如图 9(a) 所示. 除 $53 \mathrm{Ma}$ 左右格陵兰-冰岛脊靠近大陆边缘时 产生的巨大岩浆熔融通量(约 $55 \mathrm{~m}^{3} \mathrm{~s}^{-1}$ ) 外, 夏威夷和 冰岛的通量在数值和趋势上都比较一致. 与夏威夷 和冰岛热点相比, $40 \mathrm{Ma}$ 以来马里昂热点的活动强度 明显较小. 在 74 和 $50 \mathrm{Ma}$ 这两个高值时期, 马里昂热 点受到了 RTJ 和西南印度洋中脊的影响, 其活动强 度与夏威夷热点的强度在幅值上相近. 从频率域来 看(图 9(b)), 马里昂热点的活动周期明显受到了热点中脊距离的影响, 其 $25.5 \mathrm{Ma}$ 的活动周期性与 74 和 $50 \mathrm{Ma}$ 这两个岩浆通量高值相对应.

夏威夷和冰岛热点分别位于太平洋和大西洋, 但却具有相近的活动周期 (分别为 16.3 和 $15 \mathrm{Ma}$ ). Mejelde 等 ${ }^{[5]}$ 据此认为这种相近的周期反映了深部核 幔边界的大尺度波动，也就意味着地核以一个 $15 \mathrm{Ma}$ 的周期性“脉动”过程向地幔放热. 马里昂热点远离 夏威夷和冰岛热点, 应是验证这种假说的理想热点. 但我们计算的马里昂热点活动的周期 $(25.5 \mathrm{Ma}$ ) 明显比 夏威夷热点和冰岛热点活动周期(约 $15 \mathrm{Ma}$ )要长, 而 且主要与热点-洋中脊距离相关. 因此马里昂的实例表 明岩石圈的不均匀性也可导致热点活动的准周期表 现.

\section{5 转换断层的热阻断效应}

沿洋中脊的剩余地幔布格重力异常显示, 马里 昂热点的影响可以越过 $\mathrm{M}$, ES 等几何尺度较小的转 换断层, 却终止于 $\mathrm{AB}$ 和 $\mathrm{DII}$ 这两个超长的转换断 层 ${ }^{[25]}$. 据此, Georgen 等 ${ }^{[25]}$ 认为转换断层在热点沿扩 张中脊的传播中起到热阻断作用, 而且越大的转换 断层其阻断效应就越明显 ${ }^{[25]}$. 本文的板块重构表明, 在马里昂热点生成德尔卡诺隆起的这一段强烈岩浆 活动期间(73.6 42.7 Ma, 图 5(e) (h)), 马里昂热点的 位置一直位于 DII 和 IN 转换断层之间. 在向东方向, 热点沿中脊的传播(65 45 Ma)形成“V”字地形, 此地 形异常幅值在经过 GA 转换断层后明显变低, 在经过 AII 转换断层后完全消失. 在向西方向, 德尔卡诺隆 起的位置明显没有越过 DII 转换断层. 这表明 DII 和 AII 不仅阻断了现在热点沿扩张中脊的传播，而且在 整个的马里昂-西南印度洋中脊交互作用的历史中都
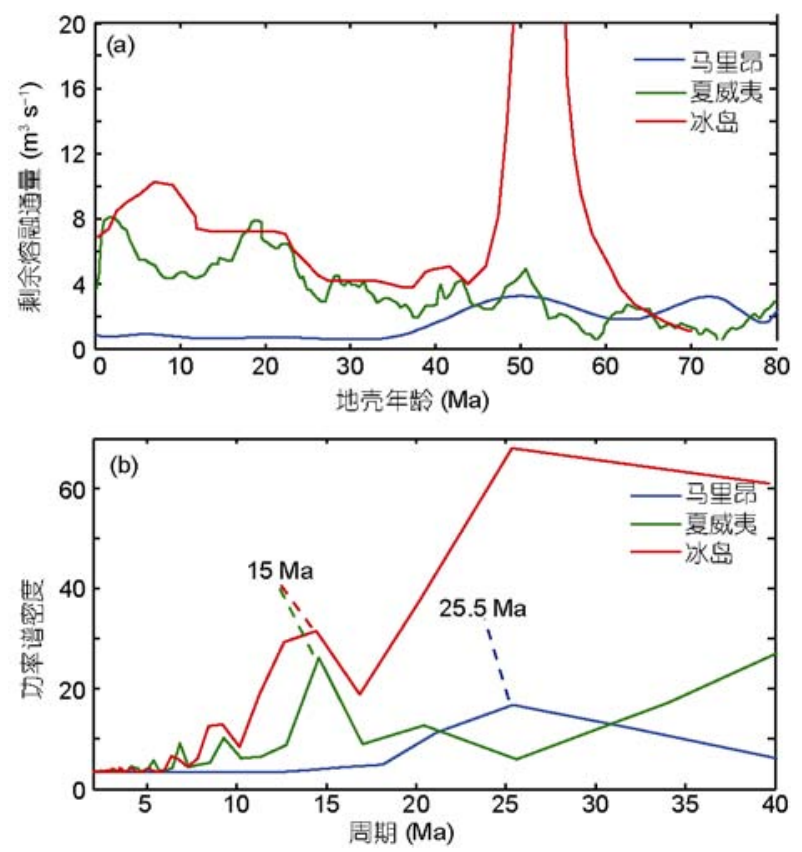

图 9 热点剩余岩浆熔融通量的对比

(a) 马里昂热点、夏威夷和冰岛热点的活动强度; (b) 马里昂热点、 夏威夷和冰岛热点的剩余岩浆通量功率谱密度

起到了非常明显的热阻断作用. 较短的转换断层如 IN 和 GA 等，虽然具有一定的热阻断效应，但是没法 完全阻断热点效应沿中脊的传播.

\section{6 克洛泽热点的起源}

由于和康拉德、凯尔盖朗(Kerguelen)热点的距离 和轨迹相近, 克洛泽热点的起源及路径充满了争议. Curray 等 ${ }^{[36]}$ 推断从拉冶马哈火成岩区(Rajmahal traps, $120 \mathrm{Ma}$ )经 $85^{\circ} \mathrm{E}$ 海岭到阿法纳西尼基丁海台(Afanasy Nikitin, $70 \mathrm{Ma}$ )是克洛泽热点在印度洋板块的轨迹, 其中拉冶马哈火成岩区是克洛泽热点形成初期生成 的大岩浆岩省, $85^{\circ} \mathrm{E}$ 海岭为热点轨迹, 阿法纳西尼基 丁海台是热点与印度-南极洲洋中脊相遇时候形成的 海台. 但是其他地球物理和地球化学证据并不支持 这种说法, Muller 等 ${ }^{[11,49]}$ 基于热点参考系的重构(本文 即采用这种方法)以及考虑热点绝对运动的板块重构 表明, $85^{\circ} \mathrm{E}$ 海岭和阿法纳西尼基丁海台都是源于现 在不再活动康拉德热点. 更多的学者认为拉冶马哈 火成岩区源于凯尔盖朗热点 ${ }^{[30,50]}$, Kent 等 ${ }^{[51]}$ 对 $\mathrm{Pb}$ 和 $\mathrm{Sr}$ 同位素的分析表明，克洛泽可能和拉冶马哈火成 岩区并无关系. Mahoney 等 ${ }^{[35]}$ 对克洛泽和阿法纳西尼 
基丁海台的 $\mathrm{Pb}$ 同位素分析表明, 两者的地球化学性 质差别非常大, 不支持克洛泽生成阿法纳西尼基丁 海台的推论.

若排除 $85^{\circ} \mathrm{E}$ 海岭, 克洛泽在印度板块(90.0 58.6 $\mathrm{Ma})$ 和南极洲板块(58.6 0 Ma) 的路径上都没有明显 海山链存在, 而作为板块边界的洋中脊也与克洛泽 热点有着不同的地球化学性质 ${ }^{[35]}$. 即便考虑热点的 绝对运动和板块重构的误差, 在研究的区域内, 也没 有任何与克洛泽相符的海山链轨迹. 这表明克洛泽 热点之前在很长的一段时间内, 甚至于从来都没有 过强烈的岩浆活动. 这种现象与 Morgan ${ }^{[2]}$ 最早提出 的地幔柱成因热点的假说并不一致, 但却与 Courtillot 等 ${ }^{[52]}$ 最近统计的很多的热点性质相似, 进 一步说明不是所有的热点都存在相应轨迹上的海山 链.

致谢法国国家研究中心(CNRS)的 Daniel Sauter 博士提供西南印度洋中脊的多波束水深数据. 山东科技大学赵俐 红博士提供热点重追踪程序. 本文很多认识得益于与中国科学院南海海洋研究所孙珍博士、美国伍兹霍尔海 洋研究所的朱健博士、北京大学王婷婷博士研究生、美国 Old Dominion 大学 Jennifer Georgen 博士以及国家 海洋局第二海洋研究所的吴招才博士、沈中延博士和杨春国博士研究生的讨论, 还有审稿人提出修改意见. 本文大部分图件使用 GMT (Wessel \&Smith, 1995)绘制. 在此一并致谢.

\section{参考文献}

1 Wessel P. Sizes and ages of seamounts using remote sensing: Implications for intraplate volcanism. Science, 1997, 277: 802-805

2 Morgan W. Rodriguez, Darwin, Amsterdam, a second type of hotspot island. J Geophys Res, 1978, 83: 5355-5360

3 Escartín J, Cannat M, Pouliquen G, et al. Crustal thickness of V-shaped ridges south of the Azores: Interaction of the Mid-Atlantic Ridge $\left(36^{\circ}-39^{\circ} \mathrm{N}\right)$ and the Azores hot spot. J Geophys Res, 2001, 106: 21719-21735

4 Van Ark E, Lin J. Time variation in igneous volume flux of the Hawaii-Emperor hot spot seamount chain. J Geophys Res, 2004, 109: B11401, doi: 10.1029/2003JB002949

5 Mjelde R, Faleide J. Variation of Icelandic and Hawaiian magmatism: Evidence for co-pulsation of mantle plumes? Mar Geophys Res, 2009, 30: $61-72$

6 Ito G, Lin J. Oceanic spreading center-hotspot interactions: Constraints from along-isochron bathymetric and gravity anomalies. Geology, 1995, 23: 657-660

7 Maia M, Goslin J, Gente P. Evolution of the accretion processes along the Mid-Atlantic Ridge north of the Azores since 5.5 Ma: An insight into the interactions between the ridge and the plume. Geochem Geophys Geosyst, 2007, 8: Q03013, doi: 10.1029/2006GC001318

8 Vidal V, Bonneville A. Variations of the Hawaiian hot spot activity revealed by variations in the magma production rate. J Geophys Res, 2004, 109: B03104, doi: 10.1029/2003JB002559

9 Adam C, Vidal V, Escartín J. 80-Myr history of buoyancy and volcanic fluxes along the trails of the Walvis and St. Helena hotspots (South Atlantic). Earth Planet Sci Lett, 2007, 261: 432-442

10 Jokat W, Ritzmann O, Schmidt-Aursch M C, et al. Geophysical evidence for reduced melt production on the Arctic ultraslow Gakkel mid-ocean ridge. Nature, 2003, 423: 962-965

11 Gente P, Dyment J, Maia M, et al. Interaction between the Mid-Atlantic Ridge and the Azores hot spot during the last 85 Myr: Emplacement and rifting of the hot spot-derived plateaus. Geochem Geophys Geosyst, 2003, 4: 8514, doi: 10.1029/2003GC000527

12 Dick H J B, Lin J, Schouten H. An ultraslow-spreading class of ocean ridge. Nature, 2003, 426: 405-412 
13 Tao C, Lin J, Guo S, et al. First discovery and investigation of a high temperature hydrothermal vent field on the ultraslow spreading Southwest Indian Ridge. EOS Trans AGU Fall Meet Suppl Abstract, 2007. T52B-07

14 Goslin J, Patriat P. Absolute and relative plate motions and hypotheses on the origin of five aseismic ridges in the Indian Ocean. Tectonophys, 1983, 101: 221-244

15 Coffin M F, Eldholm O. Large Igneous Provinces: Crustal structure, dimensions, and external consequences. Rev Geophys, 1994, 32: 1-36

16 Sinha M C, Louden K E, Parsons B. The crustal structure of the Madagascar Ridge. Geophys J Roy Astro Soc, 1981, 66: 351-377

17 Smith W H F, Sandwell D T. Global sea floor topography from satellite altimetry and ship depth soundings. Science, 1997, 277: 1956-1962

18 Müller R D, Sdrolias M, Gaina C, et al. Age, spreading rates, and spreading asymmetry of the world's ocean crust. Geochem Geophys Geosyst, 2008, 9: Q04006, doi: 10.1029/2007GC001743

19 Patriat P, Segoufin J. Reconstruction of the central Indian Ocean. Tectonophys, 1988, 155: 211-234

20 Cannat M, Rommevaux-Jestin C, Sauter D, et al. Formation of the axial relief at the very slow spreading Southwest Indian Ridge (49 ${ }^{\circ}$ to $69^{\circ}$ E). J Geophys Res, 1999, 104: 21825-21843

21 Meyzen C M, Ludden J N, Humler E, et al. New insights into the origin and distribution of the DUPAL isotope anomaly in the Indian Ocean mantle from MORB of the Southwest Indian Ridge. Geochem Geophys Geosyst, 2005, 6: Q11K11, doi: 10.1029/2005GC000979

22 Font L, Murton B J, Roberts S, et al. Variations in melt productivity and melting conditions along SWIR $\left(70^{\circ} \mathrm{E}-49^{\circ} \mathrm{E}\right)$ : Evidence from olivine-hosted and plagioclase-hosted melt inclusions. J Petrol, 2007, 48: 1471-1494

23 Mendel V, Sauter D, Rommevaux-Jestin C, et al. Magmatic-tectonic cyclicity at the ultra-slow spreading Southwest Indian Ridge: Evidence from variations of axial volcanic ridge morphology and abyssal hills pattern. Geochem Geophys Geosyst, 2003, 4: 9102, doi: $10.1029 / 2002 \mathrm{GC} 000417$

24 Sauter D, Patriat P, Rommevaux-Jestin C, et al. The Southwest Indian Ridge between $49^{\circ} 15^{\prime} \mathrm{E}$ and $57^{\circ} \mathrm{E}$ : Focused accretion and magma redistribution. Earth Planet Sci Lett, 2001, 192: 303-317

25 Georgen J E, Lin J, Dick H J B. Evidence from gravity anomalies for interactions of the Marion and Bouvet hotspots with the Southwest Indian Ridge: Effects of transform offsets. Earth Planet Sci Lett, 2001, 187: 283-300

26 Muller M R, Minshull T A, White R S. Crustal structure of the Southwest Indian Ridge at the Atlantis II Fracture Zone. J Geophys Res, 2000, 105: 25809-25828

27 Muller M R, Minshull T A, White R S. Segmentation and melt supply at the Southwest Indian Ridge. Geology, 1999, 27: 867-870

28 Minshull T A, Muller M R, White R S. Crustal structure of the Southwest Indian Ridge at $66^{\circ} \mathrm{E}$ : Seismic constraints. Geophys J Int, 2006 , 166: $135-147$

29 Goslin J, Segoufin J, Schlich R, et al. Submarine topography and shallow structure of the Madagascar Ridge, western Indian Ocean. Geol Soc Am Bull, 1980, 91: 741-753

30 Duncan R A, Richards M A. Hotspots, mantle plumes, flood basalts, and true polar wander. Rev Geophys, 1991, 29: 31-50

31 Muller R D, Royer J Y, Lawver L A. Revised plate motions relative to the hotspots from combined Atlantic and Indian Ocean hotspot tracks. Geology, 1993, 21: 275-278

32 Storey M, Mahoney J, Saunders A D, et al. Timing of hot spot-related volcanism and the breakup of Madagascar and India. Science, 1995, 267: 852-855

33 Goslin J, Diament M. Mechanical and thermal isostatic response of the Del Cano Rise and Crozet Bank (southern Indian Ocean) from altimetry data. Earth Planet Sci Lett, 1987, 84: 285-294

34 Recq M, Goslin J, Charvis P, et al. Small-scale crustal variability within an intraplate structure: The Crozet Bank (southern Indian Ocean). Geophys J Int, 1998, 134: 145-156

35 Mahoney J J, White W M, Upton B G J, et al. Beyond EM-1: Lavas from Afanasy-Nikitin Rise and the Crozet Archipelago, Indian Ocean. Geology, 1996, 24: 615-618

36 Curray J R, Munasinghe T. Origin of the Rajmahal Traps and the $85^{\circ} \mathrm{E}$ Ridge: Preliminary reconstructions of the trace of the Crozet hotspot. Geology, 1991, 19: 1237-1240

37 Divins D L. Thickness of sedimentary cover in the Eastern Pacific Ocean. In: Udintsev G B, ed. International Geological-Geophysical Atlas of the Pacific Ocean. Petersburg: Intergovernmental Oceangraphic Commission of UNESCO, 2003. 120, 126, 127, 130

38 McKenzie D. Some remarks on the development of sedimentary basins. Earth Planet Sci Lett, 1978, 40: 25-32

39 Parsons B, Sclater J. An analysis of the variation of ocean floor bathymetry and heat flow with age. J Geophys Res, 1977, 82: 802-827

40 Stein C A, Stein S. A model for the global variation in oceanic depth and heat flow with lithospheric age. Nature, 1992, 359: 123-129

41 Crough S T. The correction for sediment loading on the seafloor. J Geophys Res, 1983, 88: 6449-6454 
42 Sauter D, Cannat M, Meyzen C, et al. Propagation of a melting anomaly along the ultraslow Southwest Indian Ridge between $46^{\circ} \mathrm{E}$ and $52^{\circ} 20^{\prime} \mathrm{E}$ : Interaction with the Crozet hotspot? Geophys J Int, 2009, 179: 687-699

43 Tucholke B E, Lin J. A geological model for the structure of ridge segments in slow spreading ocean crust. J Geophys Res, 1994, 99: $11937-11958$

44 DeMets C, Gordon R, Argus D, et al. Current plate motions. Geophys J Int, 1990, 101: 425-478

45 White R S. Melt production rates in mantle plumes. Phil Trans Roy Soc London Ser A-Phys Eng Sci, 1993, 342: 137-153

46 Wessel P. An empirical method for optimal robust regional-residual separation of geophysical data. Math Geol, 1998, 30: 391-408

47 O'Neill C, Muller D, Steinberger B. Geodynamic implications of moving Indian Ocean hotspots. Earth Planet Sci Lett, 2003, 215: 151-168

48 Douglass J, Schilling J G, Kingsley R H, et al. Influence of the discovery and Shona mantle plumes on the Southern Mid-Atlantic Ridge: Rare earth evidence. Geophys Res Lett, 1995, 22: 2893-2896

49 Muller R D, Roest W R, Royer J Y. Asymmetric sea-floor spreading caused by ridge-plume interactions. Nature, 1998, 396: 455-459

50 Morgan W J. Deep mantle convection plumes and plate motions. AAPG Bull, 1972, 56: 203-213

51 Kent W, Saunders A D, Kempton P D, et al. Rajmahal basalts, eastern India: Mantle sources and melt distribution at a volcanic rifted margin. AGU Geophys Monogr, 1997, 100: 145-182

52 Courtillot V, Davaille A, Besse J, et al. Three distinct types of hotspots in the Earth's mantle. Earth Planet Sci Lett, 2003, 205: 295-308 